\title{
Reversing centrifugal forces
}

\section{Bruce Allen}

NEAR a massive compact body, such as a black hole, the centrifugal force reverses direction, according to new work by Abramowicz and coworkers ${ }^{1-3}$. The result adds another item to the list of strange relativistic effects associated with gravitationally compact objects. In addition to its intrinsic interest $^{3}$, the reversal of the centrifugal force provides a simple intuitive explanation for a number of previous predictions concerning the behaviour of viscous fluids near a black hole. These effects have important consequences for the accretion disks formed when matter, attracted by enormous gravitational forces, spirals towards such compact bodies.

The reversal of the centrifugal force can be described in terms of a simple experiment. Imagine attaching a rocket to a small test mass, and using the rocket to maintain the mass on a circular trajectory centred about a black hole. The rocket is set up so that its thrust is exerted perpendicular to the path of the test mass; thus the rotational velocity of the particle on its circular path does not change with time.

Far away from a black hole, or in empty space, the rocket must exert an inwards force, which must be increased if the rotational velocity of the test mass on its circular path is increased. If the rocket's motors are shut off, the test mass will fly off along a straight line, at a tangent to the circular path.

What Abramowicz and his collaborators have shown is that if the test mass is moving on a circular trajectory very close to a black hole, the rocket must exert an outwards force which increases if the rotational velocity of the test mass is increased. This is contrary to ordinary intuition; we would expect that increasing the rotational velocity of the test mass would tend to make the mass fly away from the black hole, so that a stronger inwardly-directed force would be needed to maintain a circular path. One can imagine building a track which circles around the black hole, and then riding a cart around the track. As the cart begins to

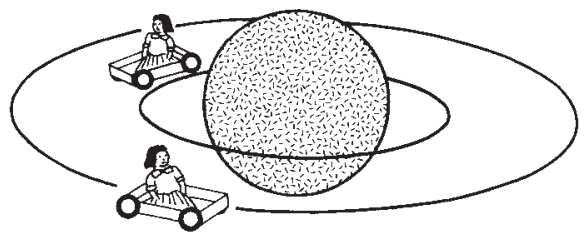

FIG. 1 A pair of circular tracks encircle a black hole, and riders in carts move around the tracks with constant velocity. The inner track lies inside a radius $3 / 2 r_{s}$, and the outer one lies outside that radius. If the riders' carts are speeded up, the outer rider is crushed more firmly against the outside of her cart; the inner rider, however, is crushed more firmly against the inside of her cart. rotate faster and faster around the track, the rider inside finds herself crushed up more and more firmly against the inside of the cart (Fig. 1).

How close does one have to be to the black hole before the centrifugal force reverses its direction? Suppose the black hole has mass $m$, and we denote the speed of light by $c$, and Newton's gravitational constant by $G$. For a compact body of mass $m$, it is convenient to introduce the 'Schwarzschild' or 'horizon' radius $r=$ $2 \mathrm{Gm} / \mathrm{c}^{2}$. For the mass of the Earth, $r$ is about a centimetre; for astrophysically interesting black holes $r$, would be at least a kilometre. The physical significance of this radius is that if the mass $m$ is collapsed into a sphere of radius $r$ smaller than $r_{s}$ a black hole forms. In that case, any object which enters within the sphere $r=r_{\text {s }}$ is doomed to fall into the gravitational singularity at $r=0$; it can never escape to the region outside the horizon.

The surprising effect found by Abramowicz and collaborators is that for circular trajectories in the equatorial plane, whose radius is less than $3 / 2 r$, the outwards force required to maintain the test mass on the circular trajectory increases with increasing rotational velocity. The roots of the idea can be found in earlier work by Abramowicz and Lasota ${ }^{+.5}$, where they show that for a test mass circling the black hole at radius $3 / 2 r$, the force required to maintain the test mass on the path is independent of its velocity. This critical radius $3 / 2 r$, at which the centrifugal force reverses sign, happens to be the same as the radius at which a photon orbits a black hole. This is no coincidence: analysis using the optical-metric formalism of Abramowicz, Carter and Lasota ${ }^{h}$ shows that, in a static space-time, the centrifugal force reverses precisely when photon geodesics (in the optical three-metric) curves inside, rather than outside, the path of the test mass. Another, equivalent statement of the effect is that the centrifugal force repels trajectories away from unstable circular photon orbits, and attracts them towards stable ones.

Fortunately, this surprising result is not a question of faith - the reader with a background in general relativity can verify the main result in an afternoon. The details can be found in section 4.1 of ref. 3; indeed, within a few years this effect will probably be presented as an exercise for students of relativity. One simply calculates the acceleration of a test mass moving with constant rotational velocity about a circular path (Fig. 2). Choosing units with $G=c=1$, one finds that the velocitydependent part of the acceleration of the test mass is perpendicular to the surfaces

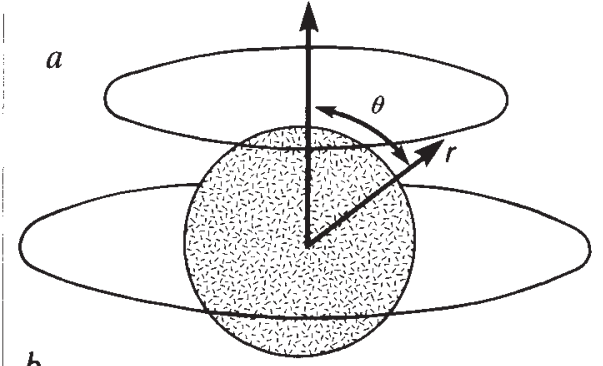

$b$

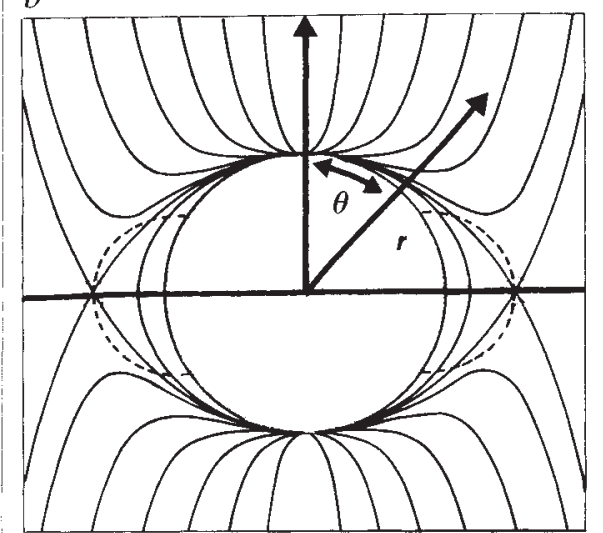

FIG, 2 Reversing centrifugal forces. Testmasses are moved in circular paths, centred around the black holes (a), which are parallel to the equatorial plane, at radius $r$ from the centre of the black hole, and an angle $\theta$ from the azimuthal axis. $b$. For a particle moving in such an orbit, the centrifugal force is normal (perpendicular) to the surfaces known as von Zeipel cylinders defined by $r^{2} \sin ^{2} \theta /(1-2 m / r)$ $=$ constant. Within the rotosphere (dashed line) the component of the centrifugal force perpendicular to the cylinders $r \sin \theta=\operatorname{con}$ stant points inwards rather than outwards.

known as von Zeipel cylinders (Fig. 2b). Inside the "rotosphere' $\left(r<3 m \sin ^{2} \theta\right)$ the component of the acceleration normal to the cylindrical surface $(r \sin \theta=$ constant $)$ points outwards rather than inwards. Thus, inside the rotosphere, the centrifugal force reverses sign.

There are a number of interesting implications of this result, and Abramowicz in fact claims that all dynamical effects associated with the centrifugal force change sign inside the rotosphere. These effects are expected to be most important for the behaviour of the accretion disks formed when matter is pulled into a black hole by gravitational forces. These accretion disks might contain a considerable quantity of material, and it is believed that they are formed when the gravitational field of the black hole tears matter off the surface of a

\footnotetext{
Abramowicz, M. A \& Prasanna A.R Mon Not $R$ astr Soc

245. 720-728 (1990).
Abramowicz, M.A. \& Miller, J.C. Mon. Not. R. astr. Soc. 245, 729-732 (1990)

3. Abramowicz, M.A. Mon. Not, R. astr. Soc. 245, 733-746 (1990)

4. Abramowicz, M.A. \& Lasota, J.-P. Acta Phys, Pol, B5 $327-329$ (1974).

5. Abramowicz, M.A. \& Lasota, J.-P. Acta Astr. 30, 35-39 (1980).

6. Abramowicz, M.A., Carter, B. \& Lasota, J.-P. Gen. Relativ. Gravitation. 20, 1173-1183 (1988)

7. Anderson, M.R. \& Lemos, J.P.S. Mon. Not. R. astr. 500 233, 489-502 (1988)

. Chandrasekhar, S. \& Miller. J.C. Mon. Not. R. astr. Soc 167, 63(1974).
} 
companion star or stars orbiting nearby.

The importance of these accretion disks stems from the simple fact that a black hole itself is black - it does not emit any light or other radiation that would make it visible from the Earth. But it might become visible indirectly as matter in the accretion disk spirals into the black hole, undergoing large accelerations and shocks, and emitting electromagnetic waves. These effects are most important near the horizon of the black hole, where the gravitational field is strongest.

To understand the behaviour of accretion disks, one needs to know, for example, how the disk transports energy and angular momentum. The reversal of the centrifugal force provides a simple explanation for several earlier results concerning the behaviour of viscous fluids near a black hole. If one spins a dinnerplate covered with syrup (a viscous fluid) the syrup spreads outwards, transporting the angular momentum outwards: but near a black hole, angular momentum is transported inwards in such a fluid ${ }^{7}$. We can now see that this is because the system attempts to minimize its energy while conserving angular momentum, reversing the direction of the viscous torque ${ }^{t}$. Another surprising result, which may also be easily understood via the reversal of the centrifugal force, is that slowly rotating contracting ellipsoids reach a maximum eccentricity, and then become more spherical as they collapse ${ }^{8}$. The Rayleigh criterion for the stability of an angular momentum distribution is similarly reversed inside the rotosphere. The hope must be that the new effect will also cast new light on the astrophysical importance of black holes, their behaviour and appearance.

Bruce Allen is in the Department of Physics, University of Wisconsin, Milwaukee, Wiscon$\sin 53211$, USA.

\section{How to find the top male}

\section{Andrew Pomiankowski}

LEKS are assemblies of males that females visit solely for the purpose of mating. An outstanding puzzle about leks is the extreme skew in the distribution of male matings - one male achieves nearly ail of the copulations, most of the others none. Several male cues are known to affect female mate choice (for example strutting rate in the sage grouse and tail length in Jackson's widowbird'); but these traits do not accurately predict the identity of the top male, nor can they alone account for the unanimity of female choice. Some other factors must be involved, and one suggestion is that females copy the mate choice of others. Wade and PruettJones ${ }^{3}$ have shown how, in theory, even a weak tendency to copy could account for the skew in male mating success, and this prediction found support from studies of grouse leks reported at a recent meeting*.

In many lek species there are several opportunities for females to copy. Females often visit in groups and make non-mating visits to the lek, when other females are present, before choosing a mate. Most theoretical models of sexual selection have ignored this, and have assumed that females choose males independently.

To study copying behaviour, Wade and Pruett-Jones draw an analogy with Polya's Urn model. In Urn models coloured balls are picked at random from an Urn and replaced with several additional balls of the same colour ${ }^{+}$. The distribution of balls

*Third international Conference of Behavioural Ecology. Uppsala, Sweden, 22-26 August 1990. after $n$ selections is given by the PolyaEggenburger equation. Copying is similar to this if the probability that a female chooses to mate with a male is dependent

\section{IMAGE UNAVAILABLE FOR COPYRIGHT REASONS}

\section{Sage grouse - strutting his stuff.}

on the number of times that he has been chosen before.

This equation shows that whenever copying occurs the variance in male mating success increases; more males have no mates and a few males obtain a large number of matings. Interestingly, this occurs even when females have no previous preferences for particular males. But if males do differ in their attractiveness to females in the first place, copying will increase differences and cause a marked skew in the distribution of matings.

At the meeting, there were reports of two new studies of mating sequence on the lek that provide evidence that females do indeed copy each others' choice. On days when large numbers of sage grouse hens visited the lek, hens were more likely to choose a male already selected by others (R. Gibson, J. Bradbury and S. Vehrencamp, University of California). Successful males had more matings than would be expected by independent choice, even when relative male attractiveness (calculated over the whole season) was controlled for - so it was not just that more attractive males had more mates. A similar pattern occurs in black grouse ( $\mathrm{J}$. Høglund, A. Lundberg, Uppsala University; R. Alatalo, Jÿvaskylä University). Males that mated more than once tended to copulate in sequence, either on the same or the following day".

Copying appears to be due to females finding males with other females close-by more attractive. In both fallow deer and sage grouse ( $\mathrm{R}$. Gibson et al.) the rate at which females enter male territories correlates with the number of females already present. Further support is provided by an experiment in which stuffed female black grouse were put on the territory of a male that had previously failed to mate ( $\mathrm{J}$. Høglund et al.). The result was an increase in the number of females that entered the territory of the less attractive male. However, the male still failed to mate with any female; so copying may attract females to inspect a male but clearly other criteria affect whether she will accept him.

There are alternative explanations for these observations. One possibility is that male attractiveness is not constant but varies from day to day, but this was ruled out in the study of sage grouse - there was an increase neither in the acoustic cues nor in the display rate on days that males copulated compared with days when they did not. Another possibility is that males increase their display rates once they have mated or if females are nearby. Females may merely be responding to the amplified display. Probably both copying and changes in male behaviour are important.

What benefits do females get from copying? If there is a cost involved in sampling and accurately assessing males, then copying may be a short cut to identifying a mate of high quality. Choice costs have not been measured in any lekking species but they undoubtedly exist.

1. Gibson, R.M. \& Bradbury, J.W. Behavl Ecol. Sociobiol. 18 177-123 (1985)

2. Andersson, S. Behavi Ecol. Sociobiol. 25, 403-410 (1989).

3. Wade, M.J. \& Pruett-Jones, S.G. Proc. natn. Acad, Scl U.S.A. 87, 5749-5753 (1990)

4. Feller, W. An Introduction to Probability Theory and its Applications Vol. 1, 3rd edn (Wiley, New York, 1968).

5. Høglund, J., Alatalo, R.V. \& Lundberg, A. Behaviour (in the press)

6. Clutton-Brock, T.H., Hiraiwa-Hasegawa, M. \& Robertson, A. Nature 340, 463-465 (1989).

7. Gibson, R.M. \& Bradbury, J.W. in Ecological Aspects of Social Evolution (eds Rubenstein, R.I. \& Wrangham, R.W.) 379-398 (Princeton Univ. Press, 1986).

8. Hamilton, W.D. Nature 228, 1218-1220 (1970). 